\title{
Las inyecciones intra-articulares con dextrosa fueron efectivas para aliviar el dolor y mejorar la funcionalidad de pacientes con disfunción témporo-mandibular
}

\author{
Intra-articular injections with dextrose were effective in relieving pain and improving functionality in patients with
}

temporomandibular dysfunction

\section{Comentado de:}

Zárate $M$, et al. J Altern Complement Med. 2020 Aug 11. doi: $10.1089 / a c m .2020 .0207$. Online ahead of print. PMID: $32780636^{1}$

\section{Objetivo}

Evaluar la eficacia de las inyecciones intra-articulares de dextrosa y lidocaína para el tratamiento de la disfunción de la articulación témporo-mandibular (ATM), comparándolas con una solución de lidocaína en agua estéril.

\section{Diseño}

Ensayo clínico aleatorizado y abierto de carácter pragmático realizado en consultorios de un Hospital Universitario.

\section{Participantes}

Fueron invitados a participar adultos con dolor o disfunción definida como la imposibilidad de abrir bien la boca, cansancio al masticar o ruidos en la articulación- de al menos $6 / 10$ puntos en la mandíbula o la cara, de más de tres meses de evolución.

\section{Intervención y comparación}

Fueron asignados a tres inyecciones ( 0,1 y 2 meses) a una de las siguientes ramas: 1) Grupo intervención (dextrosa al
$20 \%$ y lidocaína al 0,2\%: proloterapia con dextrosa intra-articular o PDIA); 2) Grupo control (agua estéril y lidocaína al 0,2\%). Luego de la tercera visita de evaluación se informó a los participantes qué estaban recibiendo y se les ofreció a todos recibir o continuar el tratamiento activo durante nueve meses adicionales.

\section{Medidas de resultado principales}

El desenlace primario fue la puntuación de una escala numérica (0 a 10 puntos) de dolor/disfunción facial y mandíbula y la proporción de pacientes que logró una mejora del $50 \%$ de su dolor y disfunción a los 0, 3 y 12 meses.

El desenlace secundario fue la apertura interincisal máxima (AIM) a los 0 y 3 meses. El análisis fue realizado por intención de tratar a través de un modelo de regresión de efectos mixtos.

\section{Resultados}

Fueron aleatorizados 29 participantes ( $47 \pm 17$ años de edad, 25 mujeres) que contribuyeron con un total de 43 articulaciones afectadas. Como lo muestra la Tabla 1 si bien comenzó a esbozarse una tendencia de respuesta diferente a los tres meses, las principales diferencias entre ambas ramas del estudio fueron observadas a los 12 meses de seguimiento.

No hubo efectos adversos en ninguno de los dos grupos.

Tabla 1. Reducción de los puntajes en la escala de dolor y de disfunción en los pacientes con disfunción de la articulación temporomandibular que recibieron proloterapia con dextrosa intra-articular y el grupo control a los 3 y 12 meses de seguimiento. Las reducciones en los puntajes de dolor y disfunción representan diversos grados de mejoría.

\begin{tabular}{|c|c|c|c|}
\hline Desenlaces & Dextrosa & Control & P-valor \\
\hline Dolor a los 3 meses, media (DE) & $2,9(2,6)$ & $4,3(2,6)$ & 0,19 \\
\hline Dolor a los 12 meses, media (DE) & $2,4(2,5)$ & $4,6(2,5)$ & 0,026 \\
\hline Disfunción a los 3 meses, media (DE) & $3,4(2,5)$ & $4,0(2,2)$ & 0,74 \\
\hline Disfunción a los 12 meses, media (DE) & $2,0(2,4)$ & $4,4(2,5)$ & 0,013 \\
\hline
\end{tabular}

\section{Conclusiones}

La PDIA resultó en una mejoría clínicamente importante y estadísticamente significativa del dolor y disfunción a los 12 meses en comparación con la inyección de lidocaína en pacientes con disfunción de la ATM moderada a severa.

Fuente de financiamiento/Conflicto de interés de los autores: La investigación fue financiada por los autores, quienes declaran no tener conflictos de intereses.

\section{Comentario}

La disfunción témporo-mandibular afecta con cierto patrón de cronicidad al $15 \%$ de los adultos ${ }^{1}$.

La proloterapia (cuyo nombre proviene del concepto de "terapia de proliferación") es un tratamiento basado en inyecciones de alguna solución irritante (que induce la proliferación) con intervalos semanales, quincenales o mensuales (en general, con un total de tres a ocho aplicaciones) en ligamentos, inserciones tendinosas y/o articulaciones enfermas ${ }^{2}$. Sus partidarios consideran que este estímulo desencadena un influjo de granulocitos, macrófagos y fibroblastos, la liberación de factores de crecimiento, y en último término, la producción y depósito de colágeno de mejor calidad que el de las estructuras enfermas, lo 
que conduciría a una reducción del dolor y a una mejoría en la funcionalidad. Sin embargo, estos mecanismos fisiopatológicos serían insuficientes para explicar su comportamiento a nivel intra-articular, para lo que se postula que además, ejercerían algún efecto condrogénico y sensorio-neural al bloquear receptores de potencial transitorio V1 (TRPV1, por sus iniciales en inglés) reduciendo el dolor neuropático ${ }^{3}$.

Si bien ya contábamos con cierta evidencia respecto de la eficacia de la proloterapia, la mayoría estaba referida a tratamientos de tendinopatías y/o de entesopatías (trocantér mayor ${ }^{4}$, aquileana, epicondilosis lateral, aductores de la cadera, fasciopatía plantar ${ }^{5}$ ), siendo más escasa la que respalda los tratamientos intra-articulares y de la disfunción temporo-mandibular en particular ${ }^{6}$. Los resultados del ensayo aleatorizado resumido fueron concordantes con los del recientemente publicado por Louw et al. ${ }^{7}$, respaldando el uso de esta terapia para la disfunción temporomandibular en los pacientes de Argentina.

\section{Conclusiones del comentador}

Los resultados de este ensayo clínico aleatorizado, realizado en Argentina, apoyan el uso de la proloterapia intra-articular con dextrosa para aliviar del dolor y mejorar la disfunción temporomandibular, en concordancia con los observados por otros investigadores.

Sergio Adrián Terrasa [ Servicio de Medicina Familiar y Comunitaria, Hospital Italiano de Buenos Aires; Departamento de Investigación, Hospital Italiano de Buenos Aires; Departamento de Salud Pública, Instituto Universitario Hospital Italiano de Buenos Aires. sergio.terrasa@hospitalitaliano.org.ar ]

Terrasa SA Las inyecciones intra-articulares con dextrosa fueron efectivas para aliviar el dolor y mejorar la funcionalidad de pacientes con disfunción témporo-mandibular. Evid Actual Pract Ambul. 2020;24(4):e002099. Comentado de: Zárate M, et al. Dextrose Prolotherapy Versus Lidocaine Injection for Temporomandibular Dysfunction: A Pragmatic Randomized Controlled Trial. J Altern Complement Med. 2020 Aug 11. doi: 10.1089/acm.2020.0207. Online ahead of print. PMID: 32780636

\section{Referencias}

1. Zarate MA, Frusso RD, Reeves KD, et al. Dextrose Prolotherapy Versus Lidocaine Injection for Temporomandibular Dysfunction: A Pragmatic Randomized Controlled Trial. J Altern Complement Med . 2020;p. 1-10. Available from: 10.1089/acm.2020.0207;https://dx.doi.org/10.1089/acm. 2020.0207.

2. Terrasa S, Agüero M, Frusso R. EOPs: Uso de la Proloterapia en pacientes con síndromes asociados a disfunción tendinosa crónica. Evid Act Pract Ambul. 2010;13(2). Available from: http://www.evidencia.org.ar/index.php/Evidencia/article/view/5803.

3. Topol GA, Podesta LA, Reeves KD, et al. Chondrogenic Effect of Intra-articular Hypertonic-Dextrose (Prolotherapy) in Severe Knee Osteoarthritis. PM R. 2016;8(11):1072-1082. Available from: 10.1016/j.pmrj.2016.03.008;https://dx.doi.org/10.1016/j.pmrj.2016.03.008.

4. Vicente-Lago I. La proloterapia mostró buenos resultados para aliviar el dolor de pacientes con síndrome de disfunción músculo tendinosa del trocánter mayor: cohorte prospectiva. Archivos de Medicina Familiar y General. 2019;16(2). Available from: https://revista.famfyg.com.ar/index.php/ AMFG/article/view/130.

5. Rabago D, Best TM, Beamsley M, et al. A Systematic Review of Prolotherapy for Chronic Musculoskeletal Pain. Clin J Sport Med. 2005;15(5):E376E376. Available from: 10.1097/01.jsm.0000173268.05318.a4;https://dx.doi.org/10.1097/01.jsm.0000173268.05318.a4

6. Nagori SA, Jose A, Gopalakrishnan V, et al. The efficacy of dextrose prolotherapy over placebo for temporomandibular joint hypermobility: A systematic review and meta-analysis. J Oral Rehabil. 2018;45(12):998-1006. Available from: 10.1111/joor.12698;https://dx.doi.org/10.1111/joor. 12698.

7. Louw WF, Reeves KD, Lam SKH, et al. Treatment of Temporomandibular Dysfunction With Hypertonic Dextrose Injection (Prolotherapy): A Randomized Controlled Trial With Long-term Partial Crossover. Mayo Clin Proc. 2019;94(5):820-832. Available from: 10.1016/j.mayocp.2018.07.023;https: //dx.doi.org/10.1016/j.mayocp.2018.07.023. 